\title{
Layer-in-Layer Hierarchical Nanostructures Fabricated by Combining Holographic Polymerization and Block Copolymer Self-Assembly
}

\author{
Michael J. Birnkrant, Christopher Y. Li, ${ }^{*}$ \\ A. J. Drexel Nanotechnology Institute and Department of Materials Science and \\ Engineering \\ Drexel University, Philadelphia, PA 19104 \\ Email:Chrisli@drexel.edu \\ Lalgudi V. Natarajan, Vincent P. Tondiglia, Richard L. Sutherland, \\ Science Applications International Corporation \\ 4031 Colonel Glenn Highway, Dayton, OH 45431 \\ Pamela F. Lloyd, \\ UES, Inc., 4401 Dayton-Xenia Rd. Dayton, OH 45432 \\ Timothy J. Bunning* \\ Email: Timothy.Bunning@wpafb.af.mil \\ Air Force Research Laboratory, Materials \& Manufacturing Directorate, Wright- \\ Patterson Air Force Base, OH 45433
}

\section{Experimental}

Materials: Block copolymer PEO-b-PCL (P3180-EOCL) [number molecular weight $\left(M_{n}\right.$ $5 \mathrm{k}-5 \mathrm{k} \mathrm{g} / \mathrm{mol})]$ with a polydispersity of 1.06 was purchased from Polymer Source Inc. The 
Block copolymer was dissolved in a commercially available thiol-ene formulation NOA 65, purchased from Norland Inc., and a UV initiator, Darocur 4265 from CIBA-GEIGY Company was added to make the homogenous mixture. NOA65 is a proprietary prepolymer of Norland products Inc. and it contains a tetrafunctional ene and a trifunctional thiol. The tetra functional ene in NOA65 contains two urethane groups per molecule. Detailed information can be found in reference S.1. The chemical structures of PEO-bPCL and the initiator Darocur 4265 are shown in Figure S1. 15 micron spacers were added to the solution before placing the mixture between two glass slides.

Grating Writing: Writing of the grating was performed using a Coherent Ar-ion laser (model Sabre Innova 10R/2) with a laser wavelength of $363.8 \mathrm{~nm}$ and an output power of 200-300 $\mathrm{mW}$. The writing geometry produces a one dimensional grating structure using a single beam configuration as shown in Figure S2. The interference pattern that arises from the internal reflection of the beam on the hypotenuse of an isosceles $90^{\circ}$ glass prism results in the formation of a grating. The cell was placed in optical contact with the prism hypotenuse using an index-matching fluid and the exposure time was typically $30 \mathrm{~s}$. The prism and the cell assembly were placed on a rotation stage to change the notch wavelength. The prism was kept at $\sim 80{ }^{\circ} \mathrm{C}$ during the grating writing.

Instrumentation: Optical characterization of the reflection grating was carried out using an ocean optics fiber spectrometer. A white light source coupled to a fiber-optic delivery system was used as the incident light. Bright-field transmission electron microscopy (BFTEM) was performed on a JEOL 2000FX TEM with an accelerating voltage of 
$120 \mathrm{kV}$. A Reichert Ultracut cryo-ultramicrotome was used to microtome the BR sample with a DiATOME $35^{\circ}$ diamond knife. Thin sections of $60 \mathrm{~nm}$ of the sample were cut and floated on the water surface and subsequently collected on a TEM copper grid. DSC experiments were performed using a Mettler Toledo DSC822e/400 equipped with an inner liquid nitrogen cooler. Approximately $1.5 \mathrm{mg}$ sample was peeled from the holographic cell and transferred to an Al DSC pan for heating and cooling tests. 2-D SAXS/WAXD experiments were carried out at the synchrotron X-ray beamline X27C at the National Synchrotron Light Source in Brookhaven National Laboratory. The zero pixel of the 2-D SAXS pattern was calibrated using Silver Behenate with the first-order scattering vector $q$ being $1.076 \mathrm{~nm}^{-1}$. The air scattering was subtracted. The X-ray beam spot was $0.1 \mathrm{~mm}$ in diameter. The X-ray beam was aligned parallel to the surface and normal directions of the sample. 2-D WAXD patterns were also recorded at room temperature in the same set-up with the sample to detector distance of $\sim 18 \mathrm{~cm}$.

\section{Reference:}

S. 1. https://www.norlandprod.com/adhesives/adhindex.tpl?category=NOA 


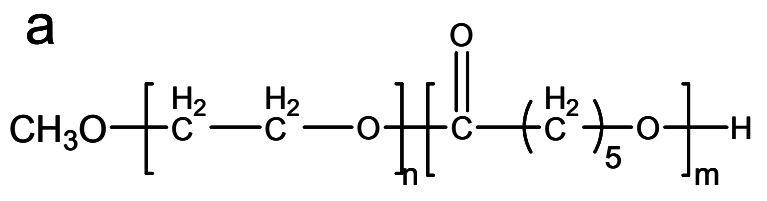

\section{Poly- $\varepsilon$-caprolactone}

b



50\% 2,4,6-Trimethylbenzoyl-diphenyl-phosphineoxide

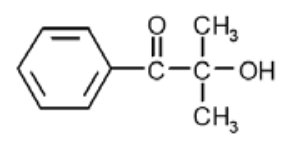

50\% 2-Hydroxy-2methyl-1-phenyl-propan-1one (Darocur 1173)

Figure S.1 Chemical Structure of (a) Poly- $\varepsilon$-caprolactone and (b) Darocur 4265, which is a mixture of the two chemicals shown in $b$. 


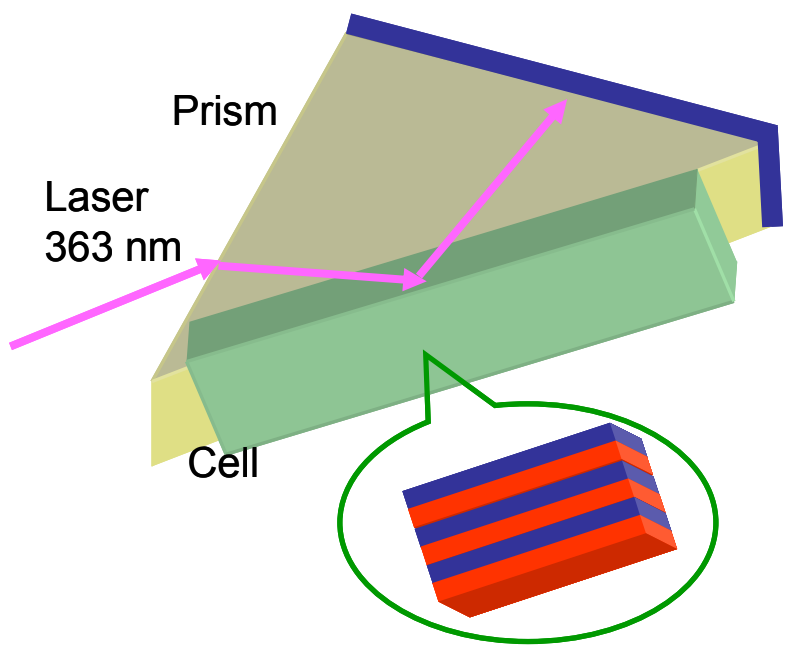

Figure S2. Experimental set-up for one-prism reflection grating. 